\title{
Auswirkungen des zweiten Shutdowns
}

Die Erholung der deutschen Wirtschaft wird durch die zweite Welle der Corona-Pandemie unterbrochen. In welchem Ausmaß die privaten und öffentlichen Maßnahmen zum Infektionsschutz die wirtschaftliche Aktivität bremsen, lässt sich anhand der konventionellen Frühindikatoren jedoch derzeit nur schwer abschätzen. So liegen Monatswerte für die Auftragseingänge und die Produktion im Verarbeitenden Gewerbe sowie für die Einzelhandelsumsätze bislang nur für den November (Shutdown light) vor. Sie deuten darauf hin, dass diese Branchen die erste Phase des Shutdowns seit Anfang November gut überstanden haben. Mitte Dezember wurden die Maßnahmen zum Infektionsschutz allerdings noch einmal verschärft. Für diesen Monat gibt es zwar schon Umfragedaten. Sie wurden jedoch zu größeren Teilen bereits vor Mitte Dezember erhoben, sodass unklar ist, inwieweit sie die wirtschaftlichen Auswirkungen abbilden. Vor diesem Hintergrund können neuere, hochfrequente Indikatoren für die wirtschaftliche Aktivität besonders nützlich sein.

Bereits zu Beginn der Corona-Pandemie im Frühjahr ist für Konjunkturanalysen verstärkt auf neue Indikatoren zurückgegriffen worden, die beispielsweise die Mobilität von Personen oder den Güterverkehr erfassen. Sie weisen in der Regel zwar einen weniger engen Zusammenhang mit der wirtschaftlichen Aktivität auf als viele der gebräuchlichen Indikatoren, welche die wirtschaftliche Aktivität zum Teil unmittelbar messen. Dafür sind diese Indikatoren aber deutlich früher, nicht selten tagesaktuell verfügbar und können somit auch kurzfristige Entwicklungen zeitnah abbilden. Ein Nachteil vieler dieser neueren Indikatoren ist jedoch, dass sie erst seit vergleichsweise kurzer Zeit zur Verfügung stehen und es dadurch erschwert wird, aus innen mithilfe üblicher Zeitreihenmodelle quantitative Prognosen abzuleiten.

Die Lkw-Fahrleistung wird seit einigen Monaten auf Tagesdatenbasis mit nur wenigen Tagen Verzögerung vom Statistischen Bundesamt ausgewiesen. Sie wird anhand von Maut-Daten berechnet und weist einen hohen Gleichlauf mit der Industrieproduktion auf. Im Frühjahr ist die Fahrleistung etwa mit Beginn des ersten Shutdowns deutlich zurückgegangen und hat damit frühzeitig Hinweise auf

(C) Der/die Autor:in(nen) 2021. Open Access: Dieser Artikel wird unter der Creative Commons Namensnennung 4.0 International Lizenz (https:// creativecommons.org/licenses/by/4.0/deed.de) veröffentlicht.

Open Access wird durch die ZBW - Leibniz-Informationszentrum Wirtschaft gefördert. das Ausmaß des wirtschaftlichen Einbruchs geliefert (vgl. Abbildung 1). Nach dem Beginn des zweiten Shutdowns im November ist keine Abschwächung bei der Fahrleistung erkennbar. Zum Jahreswechsel erschweren die großen täglichen Schwankungen die Interpretation des Indikators. Zwar ist im Sieben-Tage-Durchschnitt die Fahrleistung deutlich zurückgegangen. Dies war für diese Jahreszeit aber auch in den vergangenen Jahren zu beobachten und ist der Komplexität der Saison- und Kalenderbereinigung von Tagesdaten geschuldet. In der ersten Woche nach der Verschärfung der Maßnahmen ab dem 14. Dezember hat sich die Fahrleistung jedoch nicht abgeschwächt. Auch der starke Anstieg der Fahrleistung von mehr als $4 \% \mathrm{im}$ Dezember insgesamt spricht dafür, dass die Verschärfung der Shutdown-Maßnahmen bislang keine größeren Auswirkungen auf die Lkw-Fahrleistung hatte.

Die Mobilität von Personen kann mit verschiedenen wirtschaftlichen Aktivitäten in Zusammenhang stehen. Die Passantenzahlen in deutschen Innenstädten können vor allem für die Konsumaktivität der privaten Haushalte in Teilen des Einzelhandels sowie bei bestimmten Dienstleistungen, wie beispielsweise dem Gastgewerbe, Hinweise liefern. Allerdings weisen die täglichen Daten ein sehr starkes Saisonmuster auf, das die Interpretation erschwert. Diesem kann dadurch begegnet werden, dass man aus den jeweils in den Vorjahren beobachteten Werten unter Berücksichtigung des Wochentags, etwaiger Feiertage und der Witterungsbedingungen eine Progno-

\section{Abbildung 1}

Lkw-Maut-Fahrleistungsindex

Gleitender 7-Tage-Durchschnitt, kalender- und saisonbereinigt

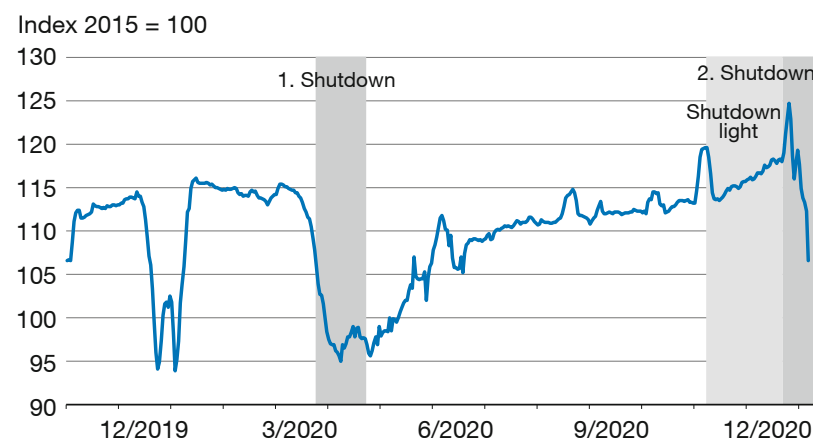

Anmerkung: Letzter Wert vom 2. Januar.

Quelle: Statistisches Bundesamt (Destatis). 


\section{Abbildung 2}

Passantenzahlen in deutschen Innenstädten

Wöchentlicher Durchschnitt, Mittel über acht Großstädte

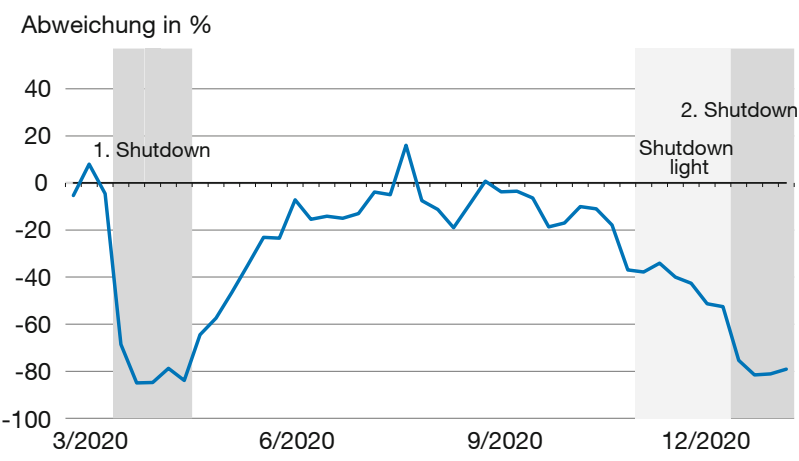

Anmerkungen: Abweichung gegenüber Prognose in \%. Letzter Wert vom 10. Januar

Quelle: hystreet.com; Corona-Datenmonitor IfW Kiel.

se erstellt und den aktuellen Wert mit diesem Referenzwert vergleicht. Auch dieser Indikator hat zu Beginn des ersten Shutdowns unmittelbar eine deutlich verminderte Passantenfrequenz in den deutschen Innenstädten angezeigt (vgl. Abbildung 2). Im Durchschnitt über mehrere Großstädte fielen die Passantenzahlen während des ersten Shutdowns um etwa $80 \%$ niedriger aus als ohne die Pandemie zu erwarten gewesen wäre. Ab Mai stiegen die Zahlen wieder deutlich an. Somit haben sie frühzeitig Signale für den Einbruch der privaten Konsumausgaben geliefert, aber auch für die anschließende Erholung. Mit dem Beginn des Shutdowns im November sind die Passantenzahlen abermals deutlich gesunken. Nach der Einführung der verschärften Maßnahmen Mitte Dezember gingen sie dann sogar wieder auf das im Frühjahr verzeichnete Niveau zurück. Dies spricht dafür, dass auch die Aktivität in den betroffenen Branchen in etwa wieder auf das Niveau vom Frühjahr zurückgefallen ist.

Ein weiterer Indikator, der täglich verfügbar ist und mit der wirtschaftlichen Aktivität in Zusammenhang steht, ist der Stromverbrauch. Gerade während der Corona-Pandemie können aber unterschiedliche Effekte auf den Stromverbrauch wirken. So könnte ein niedrigerer Stromverbrauch in der gewerblichen Wirtschaft mit einem höheren Stromverbrauch der privaten Haushalte einhergehen, wenn es zu Geschäftsschließungen oder zu vermehrten Arbeiten im Homeoffice kommt. Im Jahr 2020 verbrauchten die privaten Haushalte allerdings lediglich $26 \%$ des Stroms, während auf die Industrie $45 \%$ und auf Gewerbe, Handel und Dienstleistungen $27 \%$ entfielen. Da der Stromverbrauch kurzfristig sehr großen Schwankungen unterliegt, lässt sich die Entwicklung leichter interpretieren, wenn sie mit einem Referenzwert verglichen wird, der wiederum aus einer Prognose basierend auf den
Abbildung 3

Stromverbrauch

Wöchentlicher Durchschnitt (Werktage)

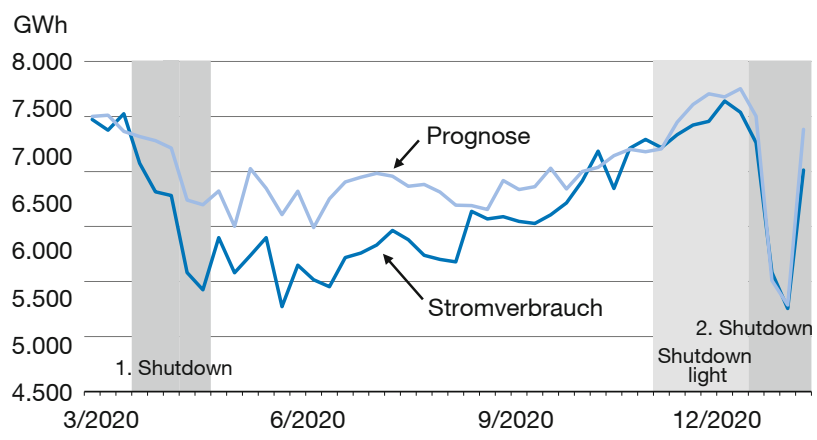

Anmerkung: Woche bis zum 10. Januar.

Quelle: ENTSO-E; Corona-Datenmonitor IfW Kiel.

Vorjahreswerten, Saison- und Kalenderfaktoren sowie den Witterungsbedingungen abgeleitet wird. Auch die Abweichung zwischen tatsächlichem Stromverbrauch und Referenzwert unterliegt kurzfristig noch größeren Schwankungen, allerdings fiel der Stromverbrauch mit Beginn des ersten Shutdowns Ende März merklich und hat so frühzeitig auf eine deutlich verminderte wirtschaftliche Aktivität hingedeutet (vgl. Abbildung 3). Im Verlauf des Novembers und Anfang 2021 ist der Stromverbrauch gegenüber dem zu erwartenden Niveau wiederum etwas zurückgeblieben. Allerdings liegt die Abweichung noch innerhalb der kurzfristigen Schwankungsbreite, die auch in der Vergangenheit zu beobachten war. Insgesamt deutet daher der Stromverbrauch bislang auf noch keinen größeren Einbruch der wirtschaftlichen Aktivität während der zweiten Corona-Welle hin.

Alles in allem sprechen die tagesaktuellen Indikatoren dafür, dass die wirtschaftlichen Auswirkungen der zweiten Corona-Welle spürbar geringer sind und sich deutlich stärker auf einzelne Branchen konzentrieren als im Frühjahr. Insbesondere für die Industrie lässt sich noch keine deutliche Abschwächung erkennen. Dieses Bild deckt sich mit den Informationen von konventionellen Frühindikatoren, wie der Industrieproduktion, den Auftragseingängen oder Umfragedaten, die bisher allerdings vor allem Auskunft für den Zeitraum vor Beginn der verschärften Shutdown-Maßnahmen liefern können. Die Wertschöpfung in Teilen des Einzelhandels und einzelnen, vor allem kontaktintensiven Dienstleistungsbranchen dürfte dagegen wieder in etwa auf das Niveau während der ersten Corona-Welle zurückfallen.

Martin Ademmer, Nils Jannsen, Hendrik Mahlkow nils.jannsen@ifw-kiel.de 